\title{
DAL-1/4.1B contributes to epithelial-mesenchymal transition via regulation of transforming growth factor- $\beta$ in lung cancer cell lines
}

\author{
FENG YU, HUA YANG, ZHANMIN ZHANG, ZHIJUN WANG and JIANPING XIONG \\ Department of Oncology, The First Affiliated Hospital of Nanchang University, Nanchang, Jiangxi 330006, P.R. China
}

Received September 15, 2014; Accepted May 8, 2015

DOI: $10.3892 / \mathrm{mmr} .2015 .4217$

\begin{abstract}
The present study aimed to investigate the effects of the tumor suppressor gene differentially expressed in adenocarcinoma of the lung 1 (DAL-1)/4.1B on early-stage adenocarcinoma of the lung. The role of DAL-1/4.1B in the epithelial-mesenchymal transition (EMT), which is implicated in cancer metastasis, was examined using DAL-1 knockdown and overexpression, followed by polymerase chain reaction and western blot analysis of EMT markers, as well as cell counting and cell migration/invasion assays. The results showed that DAL-1/4.1B has a role in transforming growth factor (TGF)- $\beta$-induced EMT in non-small cell lung cancer cells. Silencing of DAL-1/4.1B with inhibitory RNAs altered the expression of numerous EMT markers, including E-cadherin and $\beta$-catenin, whereas overexpression of DAL-1/4.1B had the opposite effect. In addition, DAL-1/4.1B expression was induced following TGF- $\beta$ treatment at the protein and mRNA level. DAL-1/4.1B deficiency impaired TGF- $\beta$-induced EMT and increased cell migration and invasion. These results suggested that DAL-1/4.1B contributed to the EMT and may be important for tumor metastasis in lung cancer. Together with the results of a previous study by our group, the present study suggested that DAL-1/4.1B acts as a tumor suppressor in the early transformation process in lung cancer, while in later stages, it functions as an oncogene affecting the biological features of human lung carcinoma cells. The results of the present study provided evidence for the feasibility of utilizing DAL-1/4.1B as a target for lung cancer gene therapy.
\end{abstract}

\section{Introduction}

Lung cancer is at present the number one cause of cancer-associated mortality of men and women. The $88 \%$-mortality rate for non-small cell lung cancer (NSCLC) has remained unchanged

Correspondence to: Dr Jianping Xiong, Department of Oncology, The First Affiliated Hospital of Nanchang University, 17 Yongwai Zheng Jie Street, Nanchang, Jiangxi 330006, P.R. China E-mail: jpxiong.jx@gmail.com

Key words: differentially expressed in adenocarcinoma of the lung 1/4.1B gene, lung carcinoma, epithelial-mesenchymal transition since 1985 despite advances in cytotoxic drug development, radiotherapy and patient management (1). An important step toward deciphering key intervention points for this disease is a clear understanding of its genetic pathobiology. Little information exists regarding the sequence of genetic events leading to the genesis of lung cancer, particularly for tumors such as adenocarcinomas, which occur in the peripheral airways of the lung. The human tumor suppressor gene differentially expressed in adenocarcinoma of the lung (DAL-1)/4.1B was identified using differential display polymerase chain reaction (DDPCR) as a gene whose expression was lacking in NSCLC when compared with matched normal tissue (2). This gene was determined to be a novel member of the Protein 4.1 superfamily by virtue of the presence of a 336 amino-acid N-terminal region with significant homology with the 4.1/Ezrin/Radixin/Moesin domain present in all 4.1 family proteins (3). Frequent loss of 4.1B in cervical cancer (4), laryngeal squamous cell carcinoma (5), breast cancer (6), esophageal squamous cell carcinoma (7) and lung adenocarcinoma (8) suggested that $4.1 \mathrm{~B}$ is a potential tumor suppressor $(9,10) .4 .1 \mathrm{~B}$ is a member of the 4.1-family of proteins together with $4.1 \mathrm{R}, 4.1 \mathrm{~N}$ and $4.1 \mathrm{G}$ and shows significant homology with ezrin, radixin and moesin as well as merlin, which is the specific gene product of neurofibromatosis type 2 . It has also been reported that loss of $4.1 \mathrm{~B}$ expression and methylation of the $4.1 \mathrm{~B}$ promoter are involved in the development and progression of NSCLC, providing a possible indicator of poor prognosis. Moreover, re-expression of protein 4.1B or a smaller fragment of the entire protein, termed DAL-1, resulted in growth suppression of meningioma cells $(11,12)$.

The epithelial-mesenchymal transition (EMT) is a biological phenomenon responsible for the formation of various tissues and organs during normal metazoan development. Due to the association of the EMT with the pathogenesis of cancer, the attention of the scientific community has been directed towards the search for and identification of effective therapeutic targets to inhibit EMT-associated phenotypic changes and tumoral progression $(13,14)$. In order to explore the role of DAL-1/4.1B in the process of EMT progression, the present study used RNA interference technology to knockdown DAL-1 expression in H460 cells and to then determine the effects on cellular proliferation and invasion. The present study attempted to elucidate the molecular mechanisms associated with the malignant progression of NSCLC, which may represent a basis for adjuvant chemotherapeutic strategies for this disease. 
Table I. Primers used in quantitative polymerase chain reaction.

\begin{tabular}{lll}
\hline Target gene & \multicolumn{1}{c}{ Forward primer } & Reverse primer \\
\hline E-cadherin & 5'-CAATGCCGCCATCGCTTAC-3' & 5'-ATGACTCCTGTGTTCCTGTTAATG-3' \\
Vimentin & 5'-GAGAACTTTGCCGTTGAAGC-3' & 5'-TCCAGCAGCTTCCTGTAGGT-3' \\
Snail1 & 5'-GCTCCACAAGCACCAAGAGT-3' & 5'-ATTCCATGGCAGTGAGAAGG-3' \\
Snail2 & 5'-CTTTTTCTTGCCCTCACTGC-3' & 5'-ACAGCAGCCAGATTCCTCAT-3' \\
Twist1 & 5'-TGCATGCATTCTCAAGAGGT-3' & 5'-GTTTTGCAGGCCAGTTTGAT-3' \\
Fibronectin & 5'-ACCAACCTACGGATGACTCG-3' & 5'-GCTCATCATCTGGCCATTTT-3' \\
$\beta$-catenin & 5'-GTACGTCCATGGGTGGGACA-3' & 5'-GGCTCCGGTACAACCTTCAACTA-3' \\
DAL-1 & 5'-GAGCTGCCAAGCGTTTATGGA-3' & 5'-CCTGCCACTATAACGAAACTTGGAA-3' \\
$\beta$-actin & 5'-AGGTCGGAGTCAACGGATTTGGTCG-3' & 5'-TGGCCAGGGGTGCTAAGCAGT-3
\end{tabular}

\section{Materials and methods}

Cell culture and reagents. The human non-small cell lung cancer cell lines NCI H460 and A549 cells were obtained from the American Type Culture Collection (Manassas, VA, USA) were cultured in RPMI-1640 medium (Gibco-BRL, Invitrogen Life Technologies, Carlsbad, CA, USA) supplemented with $10 \%$ fetal bovine serum (FBS; Hyclone, Logan, UT, USA) in the absence of antibiotics at $37^{\circ} \mathrm{C}$ in a humidified atmosphere containing $5 \% \mathrm{CO}_{2}$. The medium of actively growing cells was replenished with medium containing $10 \mathrm{ng} / \mathrm{ml}$ TGF- $\beta$ (R\&D Systems, Minneapolis, MN, USA).

Plasmid pcDNA3-DAL-1 containing a full-length DAL-1 coding region (gene ID: 23136). This plasmid was verified by DNA sequencing. The control vector pcDNA3 was purchased from Invitrogen Life Technologies (Grand Island, NY, USA). Lipofectamine ${ }^{\circledR}$ LTX \& Plus Reagent (Invitrogen Life Technologies, Carlsbad, CA, USA) was used for transfection. To obtain stable transfectants, cells seeded in 6-well plates were transfected with $2.5 \mu \mathrm{g} /$ well plasmids using $10 \mu \mathrm{l} /$ well Lipofectamine ${ }^{\circledR}$ LTX Reagent and $2.5 \mu \mathrm{l}$ /well Plus Reagent. After $6 \mathrm{~h}$ incubation in serum and antibiotic free conditions, the medium was replaced with RPMI-1640 containing 10\% FBS, and the cells were cultured for $48 \mathrm{~h}$ prior to submitting to a 2 -week selection in medium containing G418 $(600 \mu \mathrm{g} / \mathrm{ml})$.

DAL-1 shRNA and transient transfection procedure. A commercially available vector, pGPU6/GFP/Neo (Shanghai Sangon Biotech Co, Shanghai, China), was used to generate short hairpin (sh)RNA specific for DAL-1. The human DAL-1 gene-coding sequence was obtained from GenBank (http://www.ncbi.nlm.nih.gov/genbank/), where three sets of shRNA sequences targeting the human DAL-1 gene are listed: DAL-1-sh710 (5'-GCAGTGCAAAGTGATACT TCT-3'); DAL-1-sh1329 (5'-GCAAGTGGTCTGTTGATA TAT-3') and DAL-1-sh1436 (5'-GCCGGGAGAGTTTGAACA ATT-3'). A non-specific shRNA was designed as a negative control (DAL-1-shNC): (5'-CACCGTTCTCCGAACGTG TCACGTCAAGAGATTACGTGACACGTTCGGAGAATTT TTTG-3'). All shRNAs were synthesized by Shanghai ShengGong Biotechnology Co. (Shanghai, China). These oligonucleotides were synthesized and sub-cloned into the restriction sites of the vector at $22^{\circ} \mathrm{C}$ for $1 \mathrm{~h}$. $\mathrm{H} 460$ or $\mathrm{A} 549$ cells were plated in six-well plates at a density of $6 \times 10^{4}$ cells per well and incubated overnight. Cells were then transfected with DAL-1-shRNAs (2 mg plasmid in $250 \mathrm{ml}$ RPMI-1640 medium) using $5 \mathrm{ml}$ Lipofectamine ${ }^{\mathrm{TM}} 2000$ (Invitrogen Life Technologies) according to the manufacturer's instructions. Untransfected H460 cells were included as a blank control group. The success of the transfection was determined $48 \mathrm{~h}$ later by inverted fluorescence and phase-contrast microscopy (LEICA DMIL-PH1; Leica Microsystems, Wetzlar, Germany) using three randomly-selected fields of vision.

Reverse transcription quantitative (RT-q)PCR assays. Total RNA was isolated from $\mathrm{H} 460$ or A549 cells (1x10 $10^{7}$ cells per well) using TRIzol reagent (Invitrogen Life Technologies). cDNA was synthesized using 2xTaq PCR MasterMix (Tiangen Biotech Co., Ltd., Beijing, China). The 25- $\mu 1$ reaction mixture was composed of $1 \mu \mathrm{l}$ cDNA, $2 \mu \mathrm{l}$ of the upstream and downstream primers (MBI Fermentas, Vilnius, Lithuania), $12.5 \mu 1$ PCR-2X master mix (Tiangen Biotech Co., Ltd.) and $9.5 \mu \mathrm{l}$ RNase-free water (Amresco LLC, Solon, OH, USA). PCR was performed according to the manufacturer's instructions. PCR was performed using the ABI 7500 real time system (Applied Biosystems Life Technologies, Foster City, CA, USA) using SYBR Green ${ }^{\circledR}$ Premix Ex Taq (Takara Bio Inc., Tokyo, Japan). The cycling conditions included a holding step at $94^{\circ} \mathrm{C}$ for $3 \mathrm{~min}$ and 29 cycles of $94^{\circ} \mathrm{C}$ for $30 \mathrm{sec}, 55^{\circ} \mathrm{C}$ for $30 \mathrm{sec}$ and $72^{\circ} \mathrm{C}$ for $1 \mathrm{~min}$. $\beta$-actin was used as internal control. Relative quantification was performed using the $\Delta \Delta \mathrm{CT}$ method. Primer sequences of the target genes are listed in Table I.

Antibodies and western blot analysis. The following antibodies were used in the present study: Mouse monoclonal anti-4.1B (1:200; cat. no. 514386; Santa Cruz Biotechnology, Dallas, TX, USA.); mouse monoclonal anti-vimentin (1:1,000; cat. no. 3390; Cell Signaling Technology, Inc., Danvers, MA, USA); mouse monoclonal anti- $\beta$-catenin $(1: 1,000$; cat. no. 2698; Cell Signaling Technology, Inc.); mouse monoclonal anti-Snail (1:1,000; cat. no. 3895; Cell Signaling Technology, Inc.); mouse monoclonal anti-fibronectin (1:1,000; cat. no. F7387; Sigma-Aldrich, St Louis, MO, USA); mouse monoclonal anti- $\beta$-actin $(1: 10,000$; cat. no. A5441; Sigma-Aldrich). 
Cells $\left(1 \times 10^{7}\right.$ cells per well) were lysed in pre-cooled lysis buffer ( $50 \mathrm{mM}$ Tris-HCl, $150 \mathrm{mM} \mathrm{NaCl}, 1 \mathrm{mM}$ phenylmethylsulphonyl fluoride, $1 \mathrm{mM}$ EDTA, $1 \%$ NP-40, $1 \%$ sodium deoxycholate and $0.1 \%$ SDS, $\mathrm{pH}$ 7.4) and the protein content of the lysates was assessed using a Bicinchoninic Protein Assay kit (Beyotime Institute of Biotechnology, Shanghai, China). Briefly, cells were lysed using lysis buffer $[20 \mathrm{mM}$ Tris/ $\mathrm{HCl}, \mathrm{pH} 7.5$, $137 \mathrm{mM} \mathrm{NaCl}, 1 \%$ (v/v) Triton X-100, protease inhibitor cocktail (Complete $^{\mathrm{TM}}$, EDTA-free; Roche Applied Science Indianapolis, IN, USA) and a phosphatase inhibitor cocktail (PhosSTOP; Roche Applied Science)] and sonicated on ice. Bradford protein assay was used to determine the protein concentration of lysates (Bio-Rad Laboratories, Inc., Hercules, CA, USA). Lysates were heated at $70^{\circ} \mathrm{C}$ with lithium dodecyl sulfate sample buffer (Invitrogen Life Technologies) and dithiothreitol (Sigma-Aldrich) for $10 \mathrm{~min}$. Equal amounts of cell lysate $(20 \mathrm{mg}$ ) were separated by $10 \%$ SDS-PAGE and were electroblotted onto polyvinylidene fluoride membranes (Millipore, Billerica, MA, USA). Blotted membranes were blocked with 5\% skimmed milk in Tris-buffered saline containing Tween (TBST; pH 7.6) at room temperature for $1 \mathrm{~h}$. Blots were then incubated overnight at $4^{\circ} \mathrm{C}$ with the primary antibodies followed by incubation for $1 \mathrm{~h}$ at room temperature with a 1:1,000 dilution of horseradish peroxidase-conjugated goat anti-mouse monoclonal antibody (Santa Cruz Biotechnology). After incubation, membranes were washed three times each for 15 min using $0.05 \%$ TBST. Protein bands were visualized using a diaminobenzidine coloration kit and the Fusion FX7 electrochemiluminescence analysis system (Wuhan Boster Biological Technology, Wuhan, China) according to the manufacturer's instructions. Relative band intensities were detected using Image J 1.48 software (National Institutes of Health, Bethesda, MD, USA). The density of each band was normalized against that of $\beta$-actin.

Cell counting kit-8 (CCK8) assay for assessment of cell proliferation. Exponentially growing $\mathrm{H} 460$ cells were seeded into 96-well microtiter plates at a concentration of $5 \times 10^{4}$ cells per well and allowed to attach overnight prior to DAL-1 shRNA transfection. Following 24, 48, 72 and 96 h of culture, cells were incubated with $20 \mu \mathrm{l}$ CCK 8 ( $5 \mathrm{mg} / \mathrm{ml}$; Sigma-Aldrich) at $37^{\circ} \mathrm{C}$ for $4 \mathrm{~h}$ after which the medium was decanted and the reaction stopped by addition of $150 \mathrm{ml}$ dimethylsulfoxide (Sigma-Aldrich). The spectrometric absorbance of each sample was measured at $490 \mathrm{~nm}$ using an automatic microplate reader (iMark ${ }^{\mathrm{TM}}$ microplate absorbance reader; Bio-Rad Laboratories, Inc.).

Wound-healing assays. Cells were seeded into six-well plates and cultured until $>90 \%$ confluent. Three straight wounds were scratched in each well using a sterile $200-\mu l$ pipette tip. Cells were rinsed gently with phosphate-buffered saline (PBS) and $2 \mathrm{ml}$ media (without FBS or containing 10\% FBS) was added. Images were captured at 40x magnification immediately after scratching and again after $24 \mathrm{~h}$ and $48 \mathrm{~h}$. All assays were performed in triplicate and all experiments repeated three times.

Matrigel $^{T M}$ invasion assay. Transwell chambers with 8-mm pore size polycarbonate membranes (Corning-Costar, Corning, NY, USA) were used to perform the cell invasion assay. Following $24 \mathrm{~h}$ of transfection, $2 \times 10^{4}$ cells were suspended in $200 \mu \mathrm{l}$ serum-free medium and inoculated into each extracellular matrix-coated upper compartment of the 24-well plates that were pre-coated with $50 \mu \mathrm{l} 1 \mu \mathrm{g} / \mu \mathrm{l}$ Matrigel (BD Biosciences, Franklin Lakes, NJ, USA). The lower compartment of each chamber was filled with 10,000 $\mu \mathrm{l}$ RPMI- 1640 with $30 \% \mathrm{FBS}$ and incubated for $48 \mathrm{~h}$ at $37^{\circ} \mathrm{C}$ with $5 \% \mathrm{CO}_{2}$. The cells on the upper surface were then removed using cotton tips and the cells that had migrated to the lower side of the membrane were fixed with methanol for $30 \mathrm{~min}$, stained with $0.1 \%$ crystal violet (Tianjin Yixin Hengxin Chemical Co., Ltd., Tianjin, China) for $30 \mathrm{~min}$ and washed with PBS three times. The number of invaded tumor cells was calculated in five random fields at a magnification of x200, using an inverted microscope (DP70; Olympus, Tokyo, Japan) and expressed as the average number of cells/field of view.

Statistical analysis. Each experiment was performed in triplicate. Statistical analyses were performed using SPSS statistical software, version 19.0 (SPSS, Inc. Chicago, IL, USA) for Windows. Values are expressed as the mean \pm standard deviation. Analysis of variance (ANOVA) experiments (one-way, factorial and repeated-measures ANOVA), followed by the Student-Newman-Keuls test were performed, with $\mathrm{P}<0.05$ considered to indicate a statistically significant difference between values.

\section{Results}

Downregulation of DAL-1/4.1B expression effectively suppresses DAL-1/4.1B protein expression in lung cancer cells. To examine the possible roles of DAL-1/4.1B in lung cancer cells, DAL-1/4.1B was knocked down using small interfering (si)RNA. Following transfection with DAL-1/4.1B siRNA, the levels of DAL-1/4.1B mRNA were reduced compared with those of the blank control group. At $48 \mathrm{~h}$ after transfection, DAL-1/4.1B mRNA expression was effectively knocked down by sh1329, while sh710 and sh1436 had no obvious effect (Fig. 1A). In addition, western blot analysis demonstrated that DAL-1/4.1B shRNA exerted a silencing effect on DAL-1/4.1B expression in vitro (Fig. 1B). These results confirmed that sh1329 effectively interfered with DAL-1/4.1B expression.

DAL-1/4.1B shRNA promotes cell proliferation. Compared with untransfected H460 cells (blank control group), the cell proliferation significantly increased in cells transfected with DAL-1sh1329 (Fig. 1C) $(\mathrm{P}<0.05$ at 48 h). There were no significant differences in cell proliferation between the control groups ( $\mathrm{P}>0.05$; blank controls and shNC-transfected cells).

Downregulation of DAL-1/4.1B decreases the migration of lung cancer cells in vitro. The role of DAL-1/4.1B in cell migration and invasion was evaluated by a wound-healing assay and the Matrigel-based Transwell invasion assay. Cell migration is a critical step in metastasis, and the results demonstrated that DAL-1/4.1B has a critical role in the metastasic behavior of cancer cells. The effects of DAL-1/4.1B shRNA on the characteristics of lung cancer cells were examined. Transfection of DAL-1/4.1B shRNA into H460 cells resulted in increased migration capacity compared with that of the blank control, as was evident from the migration assay (Fig. 1D and E). Culture 
A

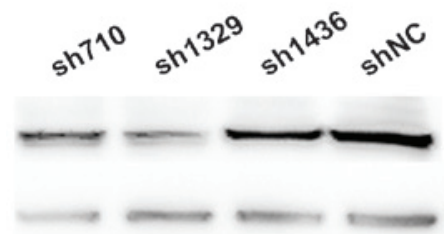

DAL-1 (108 kDa)

$\beta$-actin (42 kDa)

C

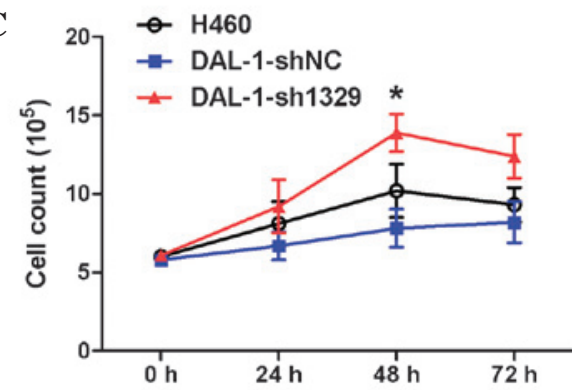

$\mathbf{E}$

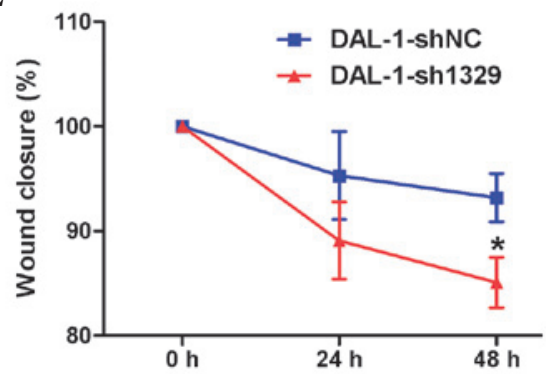

B

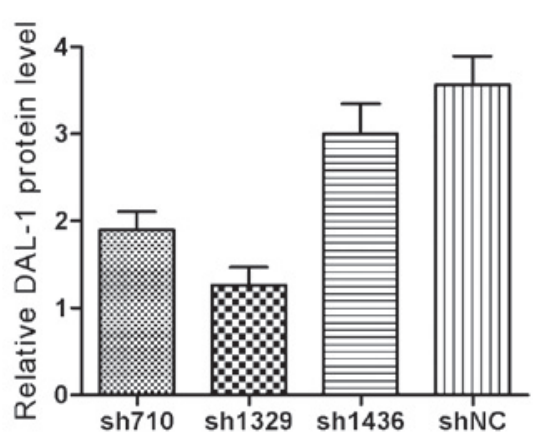

D

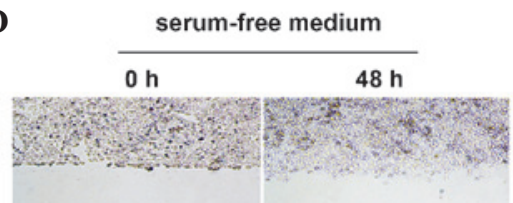

DAL-1-shNC

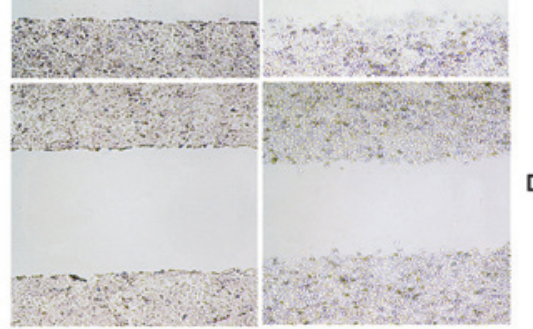

DAL-1-sh1329

Figure 1. Detection of the efficiency of DAL-1/4.1B shRNA transfection an RNA interference. (A) Representative western blot of DAL-1 protein expression in $\mathrm{H} 460$ cells $48 \mathrm{~h}$ after transfection with shRNA sequences specific for DAL-1/4.1B. DAL-1/4.1B was effectively knocked down by sh1329, while sh710 and sh1436 had no obvious effect. (B) Quantitative analysis of the relative DAL-1/4.1B protein levels as determined by western blot analysis. (C) The proliferation of $\mathrm{H} 460$ cells was assessed using a cell counting kit- 8 assay. The cell proliferation was determined by cell counting $0,24,48$ and $72 \mathrm{~h}$ following transfection. (D and E) DAL-1 knockdown attenuates cell migration. Control and DAL-1/4.1B knockdown H460 cells were subjected to a wound-healing assay in the absence of serum. Images of the cell layers were captured immediately after generation of the scratch wound and $48 \mathrm{~h}$ thereafter (magnification, $\mathrm{x} 40$ ). Values are expressed as the mean \pm standard deviation $(n=3)$. $P<0.05$ vs. DAL-1-shNC group. shRNA, small hairpin RNA; NC, negative (scrambled) control; DAL-1, differentially expressed in adenocarcinoma of the lung 1.
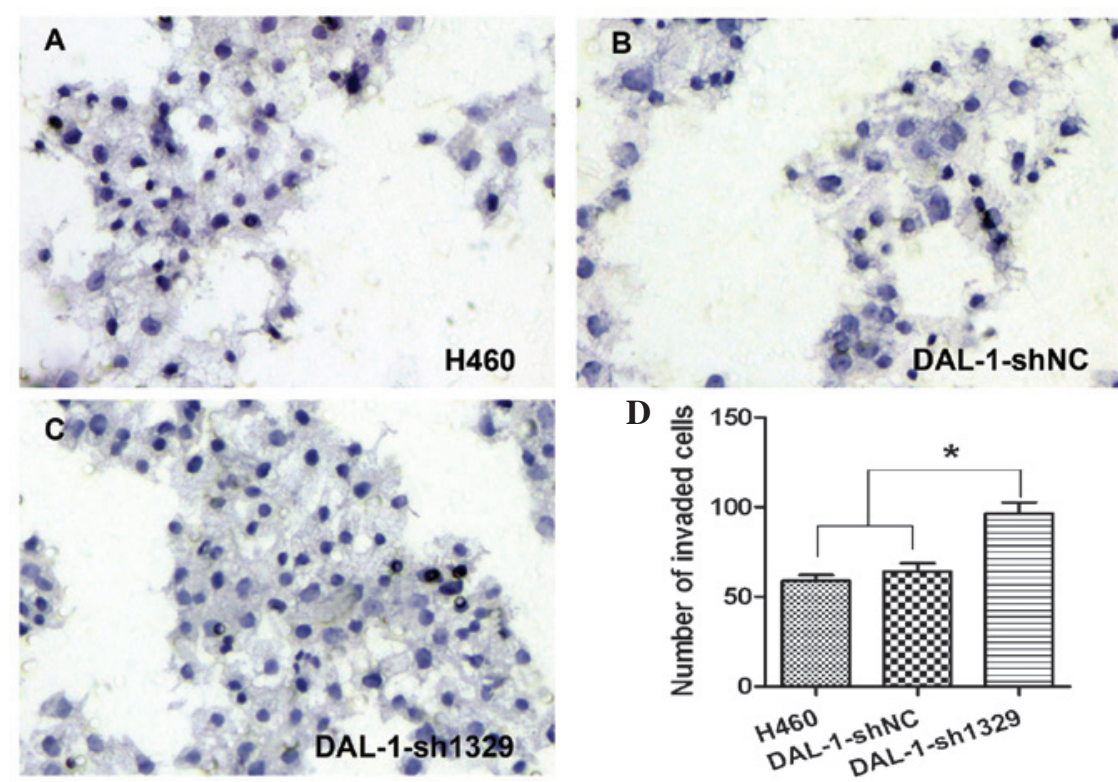

Figure 2. DAL-1/4.1B shRNA increases the invasive capacity of H460 cells. (A) H460 cells, (B) DAL-1-shNC and (C) DAL-1-sh1329 cells were seeded into the Matrigel-coated upper chambers of Transwell plates. The cells were counted under a microscope in five random fields (magnification, x200). (D) Statistical analysis showed that DAL-1 knockdown increased the invasive capacity of $\mathrm{H} 460$ cells compared with that of the control. "P<0.05, vs. H460, DAL-1-shNC and DAL-1-sh1329 cells. shRNA, small hairpin RNA; NC, negative (scrambled) control; DAL-1, differentially expressed in adenocarcinoma of the lung 1. 
A

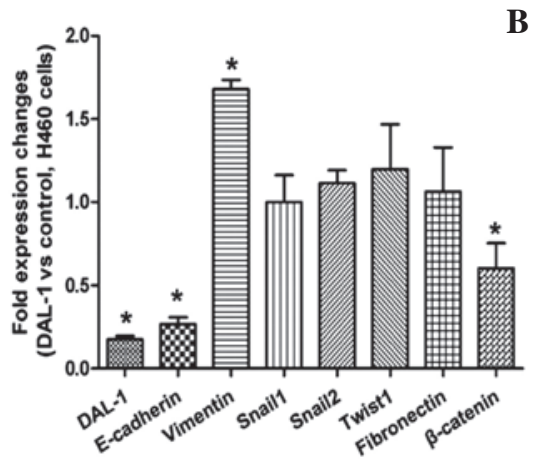

B

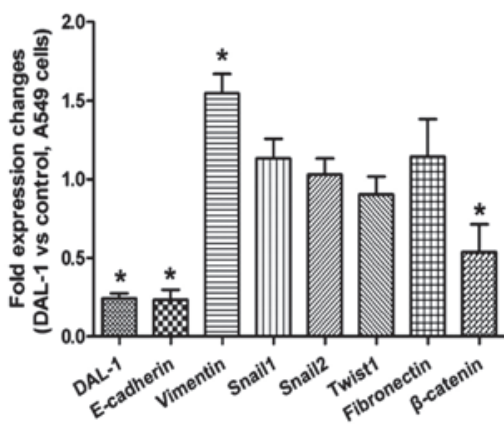

C

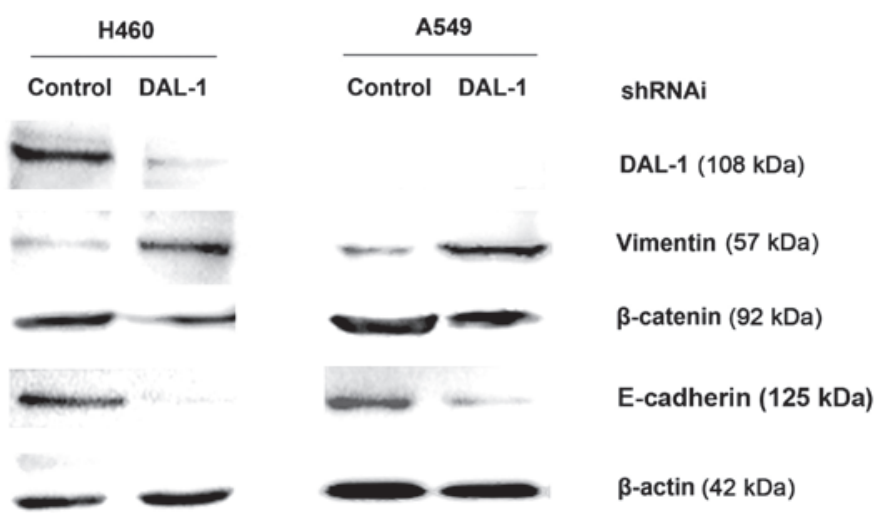

Figure 3. DAL-1/4.1B deficiency alters the expression of EMT markers. RNA extracts of control and DAL-1/4.1B-knockdown (A) A549 and (B) H460 cells were subjected to polymerase chain reaction analysis to determine the expression of target genes as indicated. The mRNA expression levels of E-cadherin and $\beta$-catenin decreased in the two cell lines, whereas the mRNA expression levels of vimentin increased. " $\mathrm{P}<0.05$ vs. control. (C) The protein expression of EMT markers was assessed in control and DAL-1/4.1B-knockdown A549 and $\mathrm{H} 460$ cells by western blotting. Representative blots show that the protein expression levels vimentin increased, while E-cadherin and $\beta$-catenin expression levels decreased. EMT, epithelial-mesenchymal transition; shRNAi, small hairpin RNA interference; DAL-1, differentially expressed in adenocarcinoma of the lung 1.

media without FBS was used to exclude the contribution of cell proliferation in the determination of migration of $\mathrm{H} 460$ cells. The average number of migrated cells in the experimental group (DAL-1-sh1329) was significantly lower than that in the blank control group (DAL-1-shNC) $(\mathrm{P}<0.05)$.

The numbers of cells which transgressed the Matrigel ${ }^{\mathrm{TM}}$ and adhered to the lower side of the membrane in the blank control (Fig. 2A), DAL-1-shNC (Fig. 2B) and DAL-1-sh1329 (Fig. 2C) groups were $59.5 \pm 6.52,64.0 \pm 8.16,96.67 \pm 10.33$, respectively. An increase in the number of invaded cells in the DAL-1-sh1329 group was observed compared with that in the control groups (Fig. 2D; $\mathrm{P}<0.05$ ), while there was no significant difference in the invasive potential between the two control groups.

$D A L-1 / 4.1 B$ deficiency alters the expression of EMT markers. To address the role of DAL-1/4.1B in H460 and A549 non-small cell lung cancer cell lines, control and DAL-1/4.1B-knockdown cells were generated by transfection with scrambled shRNA as a control and shRNA targeting DAL-1/4.1B. RT-qPCR and western blot analysis were used to examine the expression of EMT markers in control and DAL-1-knockdown H460 and A549 cells. As shown in Fig. 3A and B, E-cadherin and $\beta$-catenin mRNA were decreased, while vimentin was increased in DAL-1/4.1B-knockdown cells $(\mathrm{P}<0.05)$. However, changes in the expression of fibronectin, Snail and Twist1 following DAL-1/4.1B knockdown were not significant $(\mathrm{P}>0.05)$. Western blot analysis confirmed that DAL-1/4.1B knockdown resulted in a decrease in E-cadherin and $\beta$-catenin expression and an increase in vimentin expression at the protein level (Fig. 3C).

DAL-1/4.1B overexpression alters the expression of EMT markers. To assess the effect of DAL-1/4.1B overexpression on EMT marker expression, A549 and H460 cells stably expressing DAL-1 were generated along with control cells transfected with empty vector. As expected, DAL-1 overexpression resulted in increased E-cadherin and $\beta$-catenin expression levels, whereas vimentin levels were reduced at the mRNA as well as at the protein level (Fig. 4). Collectively, these results suggested that DAL-1 is involved in the EMT process in lung cancer cell lines.

DAL-1/4.1B expression is induced by transforming growth factor (TGF)- $\beta$. It is known that TGF- $\beta$ signaling is increased in a variety of cancer types, including lung cancer (15). To determine whether TGF- $\beta$ and DAL-1/4.1B are associated in lung cancer, the non-small cell lung cancer cell line H460, which is known to undergo EMT upon TGF- $\beta$ treatment, was utilized as a model. DAL-1/4.1B-knockdown H460 cells and scrambled control-transfected cells were incubated with TGF- $\beta$. As shown in Fig. 5A, DAL-1/4.1B protein levels were increased upon TGF- $\beta$ treatment in $\mathrm{H} 460$ cells. Although the basal levels of DAL-1/4.1B were markedly low in DAL-1/4.1B-knockdown cells, its expression was induced following incubation with TGF- $\beta$. RT-qPCR showed a similar effect on the mRNA levels of DAL-1/4.1B (Fig. 5B). 


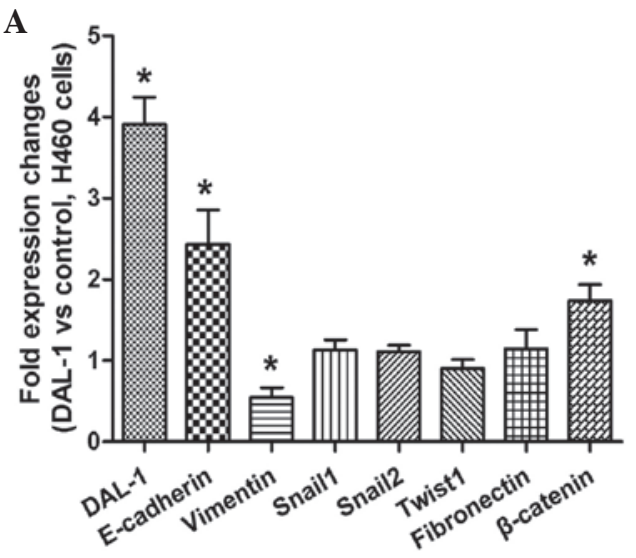

B

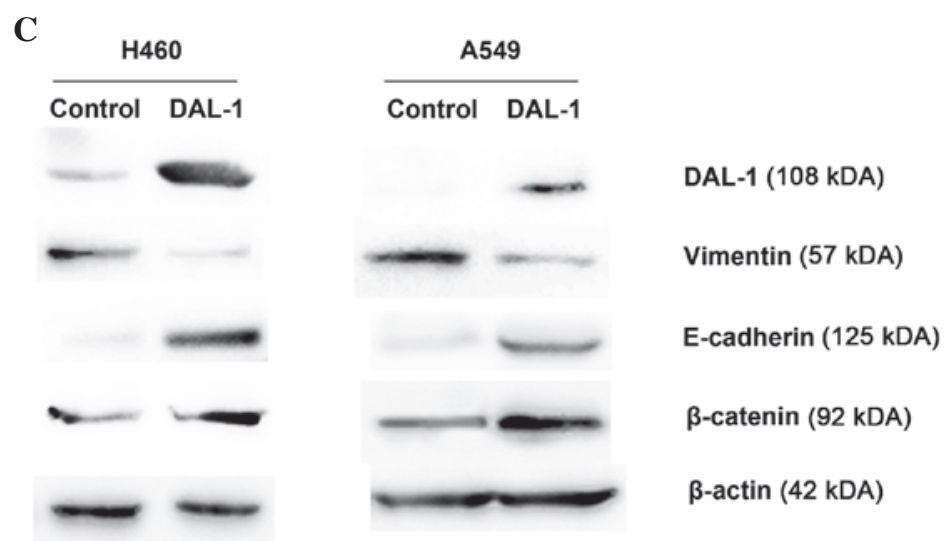

Figure 4. DAL-1/4.1B proficiency alters the expression of EMT markers. RNA extracts of (A) A549 and (B) H460 cells stably expressing control (pcDNA3) or Flag-DAL-1/4.1B were analyzed for EMT markers levels by polymerase chain reaction analysis. The mRNA expression levels of E-cadherin and $\beta$-catenin increased in the two cell lines, whereas the mRNA expression levels of vimentin decreased. ${ }^{*} \mathrm{P}<0.05$ vs. control. (C) The protein expression of target genes was determined by western blotting. Representative blots show that the protein expression levels vimentin decreased, while E-cadherin and $\beta$-catenin expression levels increased. DAL-1, differentially expressed in adenocarcinoma of the lung 1; EMT, epithelial-mesenchymal transition.

A
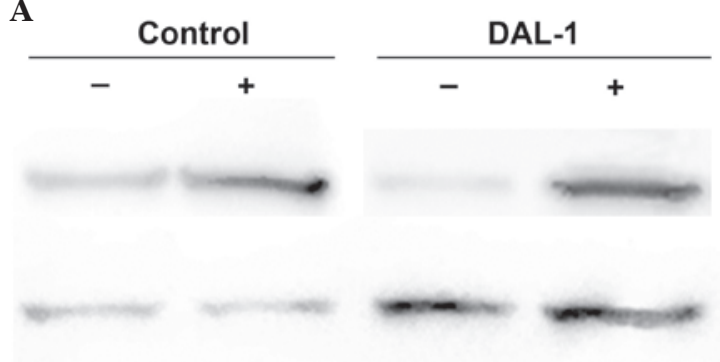

$\beta$-actin

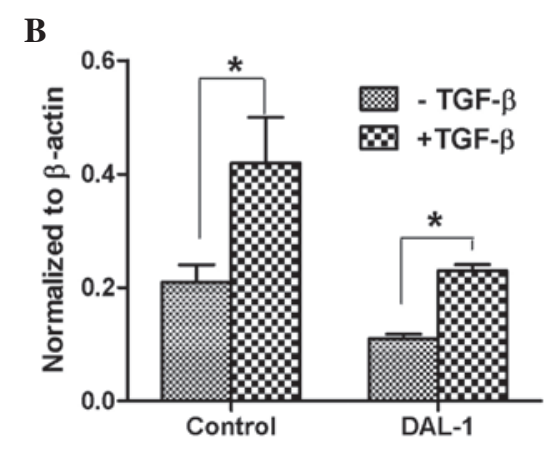

Figure 5. DAL-1/4.1B expression is induced by TGF- $\beta$. H460 stably expressing scrambled shRNA and DAL-1/4.1B shRNA were treated with TGF- $\beta(2 \mathrm{ng} / \mathrm{ml})$ for 7 days. Whole-cell lysates and RNA extracts were subjected to (A) western blotting and (B) polymerase chain reaction analysis ${ }^{*} \mathrm{P}<0.05$ vs TGF- $\beta$-untreated group. DAL- 1 , differentially expressed in adenocarcinoma of the lung 1; TGF, transforming growth factor; shRNA, small hairpin RNA.

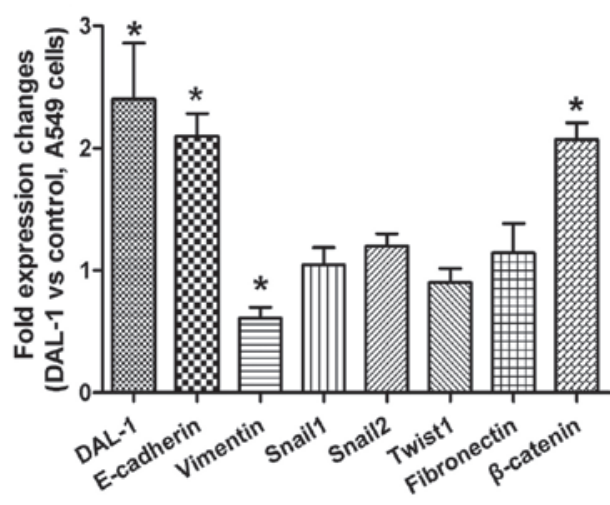

\author{
DAL-1 (108 kDA) \\ Vimentin (57 kDA) \\ E-cadherin (125 kDA) \\ $\beta$-actin (42 kDA)
}


is downregulated in several carcinoma types, including prostate cancer (21). Cavanna et al (22) identified a sub-set of genes with significantly altered expression levels between non-metastasizing and metastasizing cells in tissue culture and in primary tumors. Cells with reduced 4.1B expression displayed an altered F-actin morphology, with significantly fewer stress fibres. DAL-1/4.1B-knockdown cells migrated at twice the speed of the untreated cells. Examination of the expression of the 4.1B protein in human intestinal mucosa showed that DAL-1 was also expressed in matured epithelial cells in human colons, with a definite expression gradient along the crypt axis (23).

The EMT is characterized by a loss of cell-cell adhesion and polarity, downregulation of epithelial markers, as well as acquisition of mesenchymal markers and phenotype (24). E-cadherin, another tumor suppressor of the transmembrane adhesion molecule, also interacts with the cytoskeleton and is involved in the invasion or metastasis of gastric cancer as well as several other cancer types $(25,26)$. Accumulating evidence from studies on the EMT have indicated the involvement of numerous signaling pathways, including TGF- $\beta$, Notch, Wnt, epidermal growth factor and fibroblast growth factor $(27,28)$. Among these, TGF- $\beta$ efficiently induces EMT in a variety of model cell lines and in vivo $(29,30)$.

In conclusion, the present study showed that shRNA targeting DAL-1/4.1B significantly downregulated DAL-1/4.1B mRNA and protein expression in lung cancer cells, and inhibited cell proliferation as well as migratory and invasive potential. The results also indicated that downregulation of DAL-1/4.1B decreased the expression of E-cadherin and $\beta$-catenin in $\mathrm{H} 460$ and A549 cells. The findings of the present study provided novel insight into the underlying molecular mechanisms of NSCLC associated with the EMT, indicating that the tumor suppressor gene DAL-1/4.1B may be a potential target for anti-tumour drugs as well as gene therapy for treating lung cancer.

\section{Acknowledgements}

This study was supported by a scientific research project fund of Jiangxi Provincial Education Department (no. GJJ11374).

\section{References}

1. Sculier JP, Berghmans T and Meert AP: Small cell lung cancer: What are the treatment results in routine management? Lung Cancer 84: 101-102, 2014.

2. Tran YK, Bogler O, Gorse KM, Wieland I, Green MR and Newsham IF: A novel member of the NF2/ERM/4.1 superfamily with growth suppressing properties in lung cancer. Cancer Res 59: 35-43, 1999

3. Takakuwa Y: Protein 4.1, a multifunctional protein of the erythrocyte membrane skeleton: Structure and functions in erythrocytes and nonerythroid cells. Int J Hematol 72: 298-309, 2000.

4. Steenbergen RD, Kramer D, Braakhuis BJ, Stern PL, Verheijen RH, Meijer CJ and Snijders PJ: TSLC1 gene silencing in cervical cancer cell lines and cervical neoplasia. J Natl Cancer Inst 96: 294-305, 2004.

5. Lu B, Di W, Wang H, Ma H, Li J and Zhang Q: Tumor suppressor TSLC1 is implicated in cell proliferation, invasion and apoptosis in laryngeal squamous cell carcinoma by regulating Akt signaling pathway. Tumour Biol 33: 2007-2017, 2012.

6. Heller G, Geradts J, Ziegler B, Newsham I, Filipits M, Markis-Ritzinger EM, Kandioler D, Berger W, Stiglbauer W, Depisch D, et al: Downregulation of TSLC1 and DAL-1 expression occurs frequently in breast cancer. Breast Cancer Res Treat 103: 283-291, 2007.
7. Ito T, Shimada Y, Hashimoto Y, Kaganoi J, Kan T, Watanabe G, Murakami $\mathrm{Y}$ and Imamura M: Involvement of TSLC1 in progression of esophageal squamous cell carcinoma. Cancer Res 63: 6320-6326, 2003

8. Mao X, Seidlitz E, Truant R, Hitt M and Ghosh HP: Re-expression of TSLC1 in a non-small-cell lung cancer cell line induces apoptosis and inhibits tumor growth. Oncogene 23: 5632-5642, 2004.

9. Kikuchi S, Yamada D, Fukami T, Masuda M, Sakurai-Yageta M, Williams YN, Maruyama T, Asamura H, Matsuno Y, Onizuka M and Murakami Y: Promoter methylation of DAL-1/4.1B predicts poor prognosis in non-small cell lung cancer. Clin Cancer Res 11: 2954-2961, 2005.

10. Goto A, Niki T, Chi-Pin L, Matsubara D, Murakami Y, Funata N and Fukayama M: Loss of TSLC1 expression in lung adenocarcinoma: Relationships with histological subtypes, sex and prognostic significance. Cancer Sci 96: 480-486, 2005.

11. Gutmann DH, Hirbe AC, Huang ZY and Haipek CA: The protein 4.1 tumor suppressor, DAL-1, impairs cell motility, but regulates proliferation in a cell-type-specific fashion. Neurobiol Dis 8: 266-278, 2001.

12. Robb VA, Gerber MA, Hart-Mahon EK and Gutmann DH: Membrane localization of the U2 domain of Protein 4.1B is necessary and sufficient for meningioma growth suppression. Oncogene 24: 1946-1957, 2005.

13. Franco-Chuaire ML, Magda Carolina SC and Chuaire-Noack L: Epithelial-mesenchymal transition (EMT): Principles and clinical impact in cancer therapy. Invest Clin 54: 186-205, 2013.

14. Nieto MA: Epithelial-Mesenchymal Transitions in development and disease: Old views and new perspectives. Int J Dev Biol 53: 1541-1547, 2009

15. Elliott RL and Blobe GC: Role of transforming growth factor Beta in human cancer. J Clin Oncol 23: 2078-2093, 2005.

16. Siegel R, Naishadham D and Jemal A: Cancer statistics, 2013. CA Cancer J Clin 63: 11-30, 2013.

17. Goel A, Chhabra R, Ahmad S, Prasad AK, Parmar VS, Ghosh B and Saini N: DAMTC regulates cytoskeletal reorganization and cell motility in human lung adenocarcinoma cell line: An integrated proteomics and transcriptomics approach. Cell Death Dis 3: e 402, 2012

18. Tran Y, Benbatoul K, Gorse K, Rempel S, Futreal A, Green M and Newsham I: Novel regions of allelic deletion on chromosome 18p in tumors of the lung, brain and breast. Oncogene 17: 3499-505, 1998.

19. Yageta M, Kuramochi M, Masuda M, Fukami T, Fukuhara H, Maruyama T, Shibuya M and Murakami Y: Direct association of TSLC1 and DAL-1, two distinct tumor suppressor proteins in lung cancer. Cancer Res 62: 5129-5133, 2002.

20. Zhang Y, Xu R, Li G, Xie X, Long J and Wang H: Loss of expression of the differentially expressed in adenocarcinoma of the lung (DAL-1) protein is associated with metastasis of non-small cell lung carcinoma cells. Tumour Biol 33: 1915-1925, 2012.

21. Bernkopf DB and Williams ED: Potential role of EPB41L3 (protein 4.1B/Dal-1) as a target for treatment of advanced prostate cancer. Expert Opin Ther Targets 12: 845-853, 2008.

22. Cavanna T, Pokorna E, Vesely P, Gray C and Zicha D: Evidence for protein 4.1B acting as a metastasis suppressor. J Cell Sci 120: 606-616, 2007

23. Ohno N, Terada N, Murata S, Yamakawa H, Newsham IF, Katoh R, Ohara $\mathrm{O}$ and Ohno S: Immunolocalization of protein 4.1B/DAL-1 during neoplastic transformation of mouse and human intestinal epithelium. Histochem Cell Biol 122: 579-586, 2004.

24. Christofori G and Semb H: The role of the cell-adhesion molecule E-cadherin as a tumour-suppressor gene. Trends Biochem Sci 24: 73-76, 1999.

25. Liu Q, Mao H, Nie J, Chen W, Yang Q, Dong X and Yu X: Transforming growth factor $\{$ beta $\} 1$ induces epithelial-mesenchymal transition by activating the JNK-Smad3 pathway in rat peritoneal mesothelial cells. Perit Dial Int 28 (Suppl 3): S88-S95, 2008.

26. Xu XL, Ling ZQ, Chen SZ, Li B, Ji WH and Mao WM: The impact of E-cadherin expression on the prognosis of esophageal cancer: A meta-analysis. Dis Esophagus 27: 79-86, 2014.

27. Nakaya Y and Sheng G: EMT in developmental morphogenesis. Cancer Lett 341: 9-15, 2013.

28. Saito A: EMT and EndMT: Regulated in similar ways? J Biochem 153: 493-495, 2013.

29. Willis BC and Borok Z: TGF-beta-induced EMT: Mechanisms and implications for fibrotic lung disease. Am J Physiol Lung Cell Mol Physiol 293: L525-L534, 2007.

30. Duangkumpha K, Techasen A, Loilome W, Namwat $\mathrm{N}$, Thanan R, Khuntikeo N and Yongvanit P: BMP-7 blocks the effects of TGF-beta-induced EMT in cholangiocarcinoma. Tumour Biol 35: 9667-9676, 2014. 